J. Lake Sci. (湖泊科学), $2006, \mathbf{1 8 ( 4 ) ~ : 3 5 6 - 3 6 2 ~}$

http:// www. jlakes. org. E-mail: jlakes@ niglas. ac.cn

(c) 2006 by Journal of Lake Sciences

\title{
太湖梅梁湾水体悬浮颗粒物吸收系数的分离”
}

赵巧华 ${ }^{1,2,3}$, 张运林 ${ }^{1}$, 秦伯强 ${ }^{1 * *}$

(1: 中国科学院南京地理与湖泊研究所, 南京 210008)

(2: 中国科学院研究生院, 北京 100039)

(3: 南京信息工程大学环境科学与工程系, 南京 210044)

摘 要: 针对 2004 年 7 月 17 日梅梁湾 16 个采样点悬浮颗粒物的吸收系数,利用基于光谱标准的方法, 将悬浮颗粒物的吸 收系数分离成藻类和非藻类颗粒物两种, 并将其中藻类颗粒物的吸收系数与通过甲醇浸泡法所得的结果进行分析对比. 结果表明: 利用甲醇浸泡提取法对藻类、非藻类吸收系数的分离, 当非藻类颗粒物浓度较高时, 所得的藻类吸收系数呈现 出较明显的非藻类颗粒物的特征, 造成藻类颗粒物吸收系数有所放大, 且在短波段处体现的尤为明显; 而基于光谱标准的 模拟法能较好地将藻类颗粒物的吸收系数从总悬浮颗粒物吸收系数中分离出来, 与甲醇浸泡法相比, 藻类颗粒物在 440 、 $675 \mathrm{~nm}$ 吸收峰处的吸收系数与叶绿素 $\mathrm{a}$ 的浓度相关性 $\left(R^{2}\right)$ 得到了较为明显的提高, 分别由原来的 $0.66 、 0.75$ 提高到了 0.8964 和 0.8401 ; 就甲醇浸泡法而言, 总悬浮物吸收系数的谱形状对藻类吸收系数的放大程度有较大的影响, 当其越接 近藻类颗粒物的吸收特征时, 则色素提取法造成的误差越小, 相反, 当其越接近非藻类颗粒物的吸收特征时, 则甲醇浸泡 法造成的误差越大.

关键词: 藻类颗粒物; 非藻类颗粒物; 谱吸收系数;太湖

\section{Partitioning spectral absorption of particulate matter in Meiliang Bay of Lake Taihu}

ZHAO Qiaohua ${ }^{1,2,3}$, ZHANG Yunlin $^{1} \&$ QIN Boqiang ${ }^{1 *}$

(1:Nanjing Institute of Geography \& Limnology, Chinese Academy of Sciences, Nanjing 210008,P. R. China)

(2: Graduate School of the Chinese Academy of Sciences, Beijing 100039, P. R. China)

(3: Department of Environment Science and Engineering, Nanjing University of Information Science and Technology, Nanjing 210044 , P. R. China)

\begin{abstract}
Based on the absorption coefficients at 16 sampling sites in Meiliang Bay on July 17 2004, the absorption coefficients of algal particles were discriminated from those of nonalgal particles by the method of spectral criteria, and compared with those by methanol-extractions. The results were as follows. Discriminated from absorption coefficient of nonalgal particles by methanol-extractions, the absorption coefficient of alga particles shows the absorption characters of nonalgal particles when the concentration of nonalgal particles is high. The absorption coefficients of algal particles are overestimated especially in short wave band. By the method of spectral criteria, the absorption coefficients of nonalgal and algal particulates can be successfully separated. Compared with the partition by methanol-extractions, the limear correlation coefficients between absorption coefficient of algal particles at $440 \mathrm{~nm}$ and $675 \mathrm{~nm}$ and the concentration of chlorophyll a significantly increase. The limear correlation coefficients increase to 0.8901 and 0 . 8401 from 0.66 and 0.75 at 440 and $675 \mathrm{~nm}$, respectively. As far as the method of methanol-extractions is concerned, the spectral shape of absorption coefficient of particles matter significantly affects the degree of over-estimating the absorption coefficient of algal particles. When the spectral shape of absorption coefficient of particles matter is much more similar to that of alga particles, the error is lower; on the contrary, the error is bigger.
\end{abstract}

* 中国科学院知识创新工程项目 ( KZCX1 - SW - 12) 国家自然科学基金项目 $(40573062,40501078)$ 联合资助. 2005 - 10 - 14 收稿;2006-02-13 收修改稿. 赵巧华, 男,1972 年出生, 博士研究生. E-mail: qhzhao@ niglas. ac. cn

** 通讯作者,E-mail: qinbq@ niglas. ac. cn. 
Keywords : Algal particles; nonalgal particles; spectral absorption coefficient; Lake Taihu

水体中悬浮颗粒物的光学特性是决定水体光学特性变化的一个关键因子,一般分为两类, 其一是活体 藻类. 它通过色素捕获光能,进行光合作用, 因而它的浓度及吸收特性决定了水体的初级生产力; 其二是碎 屑, 它包含了藻类物质分解的残体, 异养的有机物 (细菌, 病毒, 浮游动物), 无机颗粒物 ${ }^{[1]}$, 其中含有非光合 作用色素 ${ }^{[2]}$. 因而将上述两类颗粒物分别称为藻类颗粒物和非藻类颗粒物 ${ }^{[3]}$. 正由于非藻类颗粒物吸收的 存在, 使得水体初级生产力计算, 藻类种群、数量变化的定量描述及二类水体的定量遥感变得更为复杂.

由于藻类颗粒物的光合作用在水体环境、生态修复等方面占有重要地位,国内外许多学者都致力于对 水体悬浮颗粒物吸收系数的分离. 随之出现了多种对藻类、非藻类吸收系数分离的方法. 其一:通过水体的 表观光学特性来推算水体中介质的吸收系数 ${ }^{[4]}$, 该方法未对藻类、非藻类吸收系数进行分离. 其二:用甲醇 提取颗粒物中的色素 (下称色素提取法), 并通过颗粒物吸收系数在提取色素前后的不同来确定藻类吸收 系数 ${ }^{[5-7]}$, 由于甲醇不仅提取了活体藻类的色素, 而且也不可避免地提取了非藻类颗粒物中的色素, 因而造 成了藻类颗粒物的吸收系数偏大, 尤其是在大型浅水内陆湖泊等二类水体中体现得更为明显, 其原因是: 在 大型浅水内陆湖泊中, 非藻类颗粒物的来源不仅有浮游植物分解的残体, 而且还有陆源有机物的残体随径 流汇人水体中, 风浪的扰动, 促使底泥再悬浮, 使得底泥中的非藻类颗粒物进人水体, 造成水体中的非藻类 颗粒物浓度较高. 其三: 根据光合色素的自身菼光特性, 利用流式细胞仪, 从物理角度将藻类颗粒物从悬浮 颗粒物中分离出来 ${ }^{[8]}$,但该方法不适用于大量水样吸收特性的分离. 其四: 基于藻类在 $675 \mathrm{~nm}$, 非藻类颗粒 在 $570 \mathrm{~nm}$ 两个波长处的吸收特性, 利用多元线性回归的方法将藻类的吸收系数分离出来 ${ }^{[14]}$, 然而该方法 假定了所有采样点的藻类光谱吸收形状是一致的,但其实际上是随种群、光照等变化而变化的. 其五: Roesler ${ }^{[12]}$ 利用脱镁叶绿素 a 在 $675 \mathrm{~nm}$ 的贡献与其浓度的关系的基础上对藻类、其他成分吸收系数进行分离. Cleveland ${ }^{[2]}$ 根据脱镁叶绿素 $\mathrm{a}$ 和脱镁叶绿 (甲酯 - ) 酸 a 在 $676 \mathrm{~nm}$ 处的吸收与二者总浓度对藻类颗粒物和 非藻类颗粒物吸收系数进行分离. Chang 和 Dickey ${ }^{[15]}$ 利用 Roesler 提出的方法将水中介质的总吸收系数(不 包括水本身) 较为成功地分离黄质、藻类颗粒物和非藻类颗粒物的吸收系数. 上述的几种数值模拟分离法 均建立在特定波长的吸收特征基础上,克服了色素提取法的不足.

本文所研究的区域是在太湖梅梁湾, 其位于太湖北部, 地处无锡市郊,陆源非藻类颗粒物较丰富. 该湾 面积约 $132 \mathrm{~km}^{2}$, 平均深度仅为 $2 \mathrm{~m}$, 因而风浪的扰动将造成底泥中非藻类颗粒物释放于水体中. 同时该处也 是一个富营养化、水华频发的区域,因而该处悬浮颗粒物的吸收特性较为复杂, 从而造成其生物光学特性有 别于开阔大洋, 也不同于近海海域. 然而在研究其初级生产率, 藻类种群变化, 水华爆发的可能机制中, 藻类 颗粒物吸收特性的准确定量却又显得尤为重要. 本文试图用基于光谱标准的方法 (下称模拟法), 将藻类的 吸收系数从悬浮颗粒物中分离出来, 以探究梅梁湾藻类光谱吸收特性. 为研究水华爆发机制、浮游植物种群 和数量的变化、生物 - 光学模型、二类水体的水质定量遥感等奠定坚实的基础.

\section{1 材料及方法}

\section{1 材料}

针对太湖梅梁湾的 16 个采样点采集的水样,样品采集后放人冷藏箱内保存, 当天带回实验室进行过滤 处理, 然后放人冰箱内冷藏, 叶绿素 $\mathrm{a}$ 及脱镁叶绿素 a 浓度的测定在 $2-3 \mathrm{~d}$ 内完成; 其中总悬浮颗粒物的吸 收系数是利用定量滤膜技术 ${ }^{[16]}$ 获取的.

\section{2 基本原理和方法}

活体藻类在 $380 \mathrm{~nm}$ 和 $505 \mathrm{~nm}$ 的吸收系数之比接近于 1 , 而且基本上为一恒定常数; 在 $580 \mathrm{~nm}$ 和 700 $\mathrm{nm}$ 处之比也基本为一常数, 从这一现象, 可得出: (1)总悬浮颗粒物的吸收系数 $\left(a_{p}\right)$ 随波长减小而逐渐增大, 主要归因于非藻类颗粒物的吸收; (2)基于 $a_{p}$ 光谱斜率所包含的信息, 藻类和非藻类颗粒的吸收系数是可以 分离出来的 ${ }^{[3]}$.

根据 Bricaud 等 $^{[3]}$ 的分析,选择藻类颗粒物的吸收系数 $\left(a_{p h}\right)$ 在不同波长之比应遵循以下三个基本原 则: (1)附属色素对所选波长处的吸收系数影响最小;(2)活体藻类在所选波长处的吸收系数之比应接近于 1; 
(3)所选的两个波长应相隔足够大, 以使计算 $a_{p}$ 的斜率有足够的精度. 同时选择不同两个波长的吸收系数之 比, 也避免了包裹效应对吸收系数的影响. 在充分考虑上述原因的基础上, Bricaud 等 $^{[3]}$ 选定了四个波长:

$$
\begin{aligned}
& a_{p h}(505): a_{p h}(380)=0.99 \\
& a_{p h}(580): a_{p h}(692.5)=0.92
\end{aligned}
$$

非藻类颗粒物的吸收系数随波长呈负指数衰减的规律, 因而采用下式中的形式:

$$
a_{d}(\lambda)=A \exp (-s \lambda)
$$

其中, $a_{d}$ 是非藻类颗粒物的吸收系数, $\lambda$ 是波长, $A 、 s$ 是变量. 同时由于总悬浮颗粒物的吸收系数是 $a_{d}$ 和 $a_{p h}$ 之和: 于是通过下面两式来确定 $A 、 s$ 两个变量.

$$
\begin{aligned}
& 0.99 A \exp (-380 s)-A \exp (-505 s)=0.99 a_{p}(380)-a_{p}(505) \\
& A \exp (-580 s)-0.92 A \exp (-692.5 s)=a_{p}(580)-0.95 a_{p}(692.5)
\end{aligned}
$$

对于 (4)、(5) 两式, 可以通过二分法确定 $A 、 s$, 然后计算出藻类和非藻类颗粒物在各个波长处的吸收系数 $\left(a_{p h}(\lambda) 、 a_{d}(\lambda)\right)$.

$$
\begin{aligned}
& a_{d}(\lambda)=A \exp (-s \lambda)+a_{p}\left(\lambda_{0}\right)-A \exp \left(-\lambda_{0} s\right) \\
& a_{p h}(\lambda)=a_{p}(\lambda)-a_{d}(\lambda)
\end{aligned}
$$

然而藻类颗粒物的吸收系数在 $700 \mathrm{~nm}$ 以后随波长增大衰减较快, 当其衰减到一定程度时, 总悬浮颗粒 物的吸收系数主要是受到非藻类颗粒物的影响, 即 $a_{p}$ 在 $\lambda_{0}$ 处的吸收主要是非藻类颗粒物贡献的, 而藻类 颗粒物的吸收贡献基本上是可以忽略, 而在 (1) 式到 (5) 式中, 非藻类颗粒物的吸收系数主要是通过两对波 长处的吸收特性分离而获得的, 因而需要对非藻类颗粒物的吸收系数进行修正. $a_{p}\left(\lambda_{0}\right)-A \exp \left(-\lambda_{0} s\right)$ 表示 的是参考总悬浮颗粒物在 $\lambda_{0}$ 处的吸收系数, 对非藻类颗粒物的吸收系数进行修正, 通过 $a_{d}(\lambda)$ 上下平移, 使得 $a_{d}\left(\lambda_{0}\right)$ 等于 $a_{p}\left(\lambda_{0}\right)^{[3]}$. 考虑到 Bricaud 等 ${ }^{[3]}$ 的研究对象是海洋, 在海洋中, 非藻类颗粒物的来源基本 上是藻类分解产品, 且其含量与内陆湖泊相比, 浓度甚低, 这种状况在文献 ${ }^{[3]}$ 中图 $8 \mathrm{a} 、$ 图 $4 \mathrm{~b}$ 中均体现出来 了, 图 8a 描述的是海洋表层总悬浮颗粒物谱吸收特征, 从中可以发现其完全是藻类的吸收特征, 既使在海 洋 $100 \mathrm{~m} 、 200 \mathrm{~m} 、 400 \mathrm{~m}$ 的深处, 其仍表示出了明显的藻类吸收特征, 因而可以得出在其研究对象中, 有机悬 浮颗粒物在悬浮物中占有绝对的比重. 所以他们选取 $\lambda_{0}$ 为 $750 \mathrm{~nm}$, 且模拟效果较好.

而我们的研究区域系一典型的大型浅水湖泊,非藻类颗粒物来源广泛,浓度甚高,因而在 $\lambda_{0}$ 为更小时, 非藻类颗粒物就成为了总悬浮颗粒物吸收系数的主要决定者. 根据 Roesler 和 Perry ${ }^{[12]}$ 的研究, 叶绿素 a 和 脱镁叶绿素 $\mathrm{a}$ 的浓度在一定程度上代表了藻类颗粒物和非藻类颗粒物的浓度. 于是采用在常规观测中叶绿 素 $\mathrm{a}$ 的浓度与脱镁叶绿素 $\mathrm{a}$ 的浓度之比,给出了一个关于 $\lambda_{0}$ 选取的关系式.

$$
\lambda_{0}=-3.45 \times \frac{[\text { pheo }]}{[\text { chla }]}+715.382
$$

其中 $[$ pheo $]$ 为脱镁叶绿素 $\mathrm{a}$ 的浓度, [chla $]$ 为叶绿素 $\mathrm{a}$ 的浓度, 上式中的 $\lambda_{0}$ 是通过 $(8)$ 式计算后, 经四舍五 人确定一个整数波长.

\section{3 活体藻类在绿光和紫光波段、黄光和红光波段的吸收比变化的影响}

Bricaud 等 $^{[3]}$ 认为 $a_{p h}(505) ： a_{p h}(380), a_{p h}(580) ： a_{p h}(592)$ 基本为一常数, 分别是 $0.99,0.92$. 然而这 两个比例可能会随区域的不同, 藻类优势种的不同而发生一定的变化, 同时他们给出了判断 (1)、(2)两式 是否适用的标准, 即如果 $s$ 接近于 0 或 $s \geqslant 0.02$ 时, 则应用(1)、(2) 两式就可能给结果带来一定的影响.

表 1 梅梁湾 16 个点 $A 、 s$ 的值

Tab. 1 The values of $A$ and $s$ of 16 sampling sites in Meiliang Bay

\begin{tabular}{ccccccccc}
\hline 点号 & $01^{\#}$ & $02^{\#}$ & $03^{\#}$ & $04^{\#}$ & $05^{\#}$ & $06^{\#}$ & $07^{\#}$ & $08^{\#}$ \\
\hline$A$ & 2536.751 & 3653.487 & 3681.191 & 3678.023 & 3184.195 & 3335.732 & 2732.586 & 1806.407 \\
$s$ & 0.01378 & 0.01447 & 0.01439 & 0.01405 & 0.01359 & 0.01438 & 0.01381 & 0.01508 \\
\hline 点号 & $09^{\#}$ & $10^{\#}$ & $11^{\#}$ & $12^{\#}$ & $13^{\#}$ & $14^{\#}$ & $15^{\#}$ & $16^{\#}$ \\
\hline$A$ & 2066.407 & 2850.220 & 3697.509 & 3959.719 & 1658.961 & 2750.339 & 2147.212 & 3757.503 \\
$s$ & 0.01512 & 0.01426 & 0.01492 & 0.01545 & 0.01333 & 0.01455 & 0.01602 & 0.01581 \\
\hline
\end{tabular}


从上表中,我们可以看出:对梅梁湾 16 个点的数据进行分析计算,结果 (表 1 ) 发现 $s$ 的值均落在 0.013 -0.01602 的范围内, 可见上述两个比例变化不大,该方法完全适用于梅梁湾的实际情况.

\section{2 结果与分析}

\section{1 对比分析}

图 1 给出了用色素提取法、模拟法得到的 $05^{\#}$ 、 $14^{\#}$ 点的藻类颗粒物的吸收系数. 就用色素提取法得到的 藻类吸收系数而言,这两个点均表示出了共同的特点:其一,在 $675 \mathrm{~nm}$ 左右有一个吸收峰,这是典型的叶绿 素 $\mathrm{a}$ 的吸收特点; 其二, 在 520 - 650 nm 之间, 其间的吸收系数变化不大; 其三,随波长的进一步减小, 其吸 收系数却基本上在持续增大. 而用模拟法所得的结果与色素提取法的结果相比, 可以看出:在 $520-700 \mathrm{~nm}$ 之间, 两个点的藻类吸收系数基本上表示出相同的形状特征, 只是后者在数值上相对较小, 且在 $520-650$ $\mathrm{nm}$ 之间, 用模拟法的结果比色素提取法的结果小得多点; 但在 520-400 nm 之间, 却表现出较大的不同: 形 状不同, 用模拟法所得的两个点的结果均在 $430 \mathrm{~nm}$ 左右有一明显的峰值; 且在数值上也比色素提取法的小 得多.

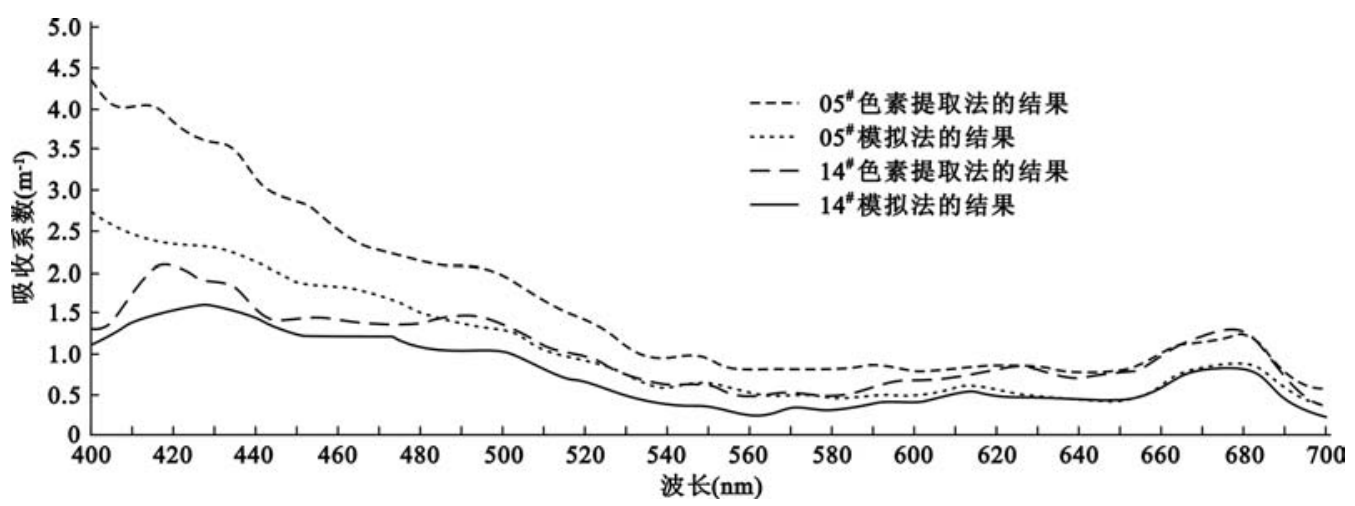

图 1 藻类颗粒物吸收系数

Fig. 1 The absorption coefficients of algal particles

根据曹文熙等 ${ }^{[6]}$ 、Gallegos 和 Neals ${ }^{[9]}$ 、Bricaud 和 Stranmski ${ }^{[3]}$ 、Neveux 和 Andre ${ }^{[10]}$ 等许多学者的研究,活 体藻类的吸收系数均在 $430 \mathrm{~nm}$ 左右有一个最大的峰值, 而不是随波长的减小持续增大的. 非藻类颗粒物 包含浮游植物的分解残体、底泥和土壤中输送腐殖物质、无机颗粒物, 而这些颗粒物吸附溶解的腐殖物质, 其吸收特征基本呈现出与黄质的类似, 即随波长的减小, 吸收系数呈指数增加 ${ }^{[11]}$. 因此用色素提取法所得 的藻类吸收系数在 $400-520 \mathrm{~nm}$ 之间的特点具有明显的非藻类颗粒物的吸收特点. 其主要原因是由于用色 素提取法时, 将非藻类中的多种色素一并提取, 从而系统地放大了藻类颗粒物的吸收系数, 这种现象在 Cleveland 和 Perry ${ }^{[2]}$ 、Bricaud 和 Stranmski ${ }^{[3]}$ 、Roesler 和 Perry ${ }^{[12]}$ 的论文中均有论述. 而且由于大型浅水湖泊 中, 非藻类颗粒物丰富, 因而该方法对藻类颗粒物吸收作用的放大现象尤为突出. 而在大于 $520 \mathrm{~nm}$ 的波长 之处, 由于非藻类颗粒物的吸收随波长的衰减, 其与藻类的吸收系数相比, 所占份额较小, 因而两种方法所 得的吸收系数差别较小, 尤其是在 $650-700 \mathrm{~nm}$ 之间, 由于在 $675 \mathrm{~nm}$ 处有一个明显的吸收峰, 非藻类颗粒物 的吸收与之相比就显得更小,所以用两种方法得到的结果差别不大.

藻类吸收系数的谱变化主要是由藻类的组成及其色素浓度的变化决定的 ${ }^{[7,12]}$, 针对藻类吸收系数的峰 值分布来看 (图 1), 其具有较为明显的叶绿素 $\mathrm{a}$ 的吸收特征. 而且国内外许多学者 ${ }^{[6,7,13]}$ 均探讨了 $a_{p h}$ (675)、 $a_{p h}(440)$ 与叶绿素 $\mathrm{a}$ 的关系. 张运林 ${ }^{[7]}$ 利用了本次采样所测得的总悬浮颗粒物系数,探究了通过色 素提取法获取的藻类颗粒物的吸收系数与叶绿素 $\mathrm{a}$ 的关系, 发现 $a_{p h}(675) 、 a_{p h}(440)$ 与叶绿素 a 均存在较 好的关系. 其相关系数 $\left(R^{2}\right)$ 分别是 $0.75 、 0.66$, 并认为藻类吸收系数在 $440 \mathrm{~nm}$ 处的吸收系数受到了非藻类 颗粒物吸收的影响大于 $675 \mathrm{~nm}$ 处. 

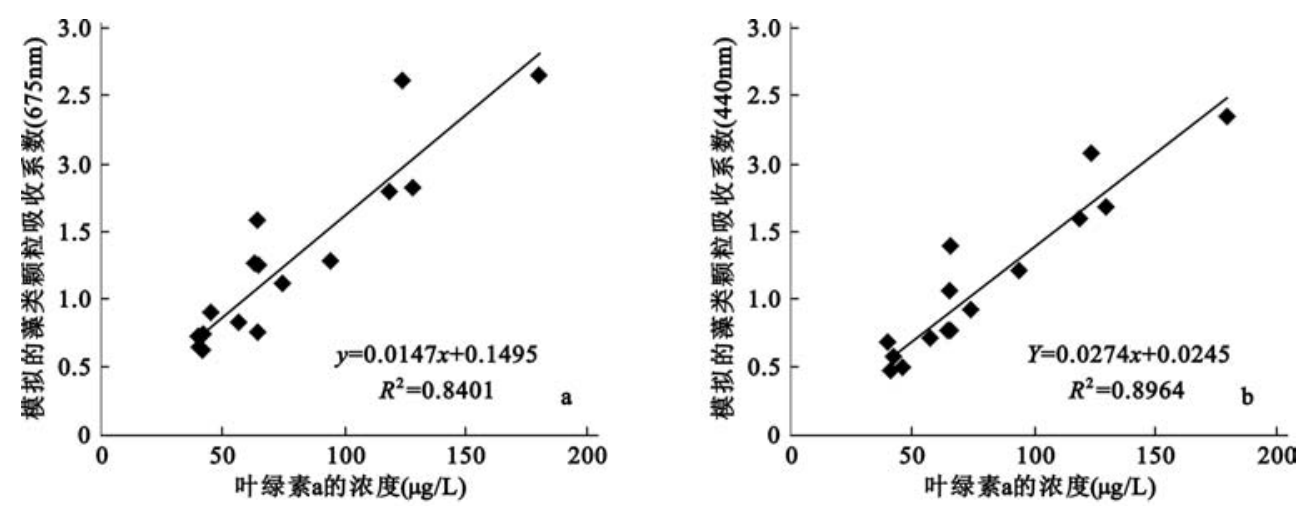

图 2 藻类吸收系数 $a_{p h}(675)$ 与叶绿素 $\mathrm{a}$ 的关系 (a) 及 $a_{p h}(440)$ 与叶绿素 $\mathrm{a}$ 的关系 (b)

Fig. 2 Correlations between $a_{p h}(675), a_{p h}(440)$ and chlorophyll a concentration

图 2 表示的是通过模拟法所得的藻类颗粒物吸收系数与叶绿素 $\mathrm{a}$ 的关系. 从中可以看出两者与叶绿素 $\mathrm{a}$ 的相关性均得到了较大的提高, $R^{2}$ 分别为 0.8401 和 0.8964 . 而且两组的相关程度也基本相当,这不仅证 实了张运林 ${ }^{[7]}$ 的判断, 而且也证实了模拟法确实可以避免藻类吸收系数的系统放大, 真实地反映了藻类的 吸收状况.

\section{2 不同谱形状的吸收系数分离}

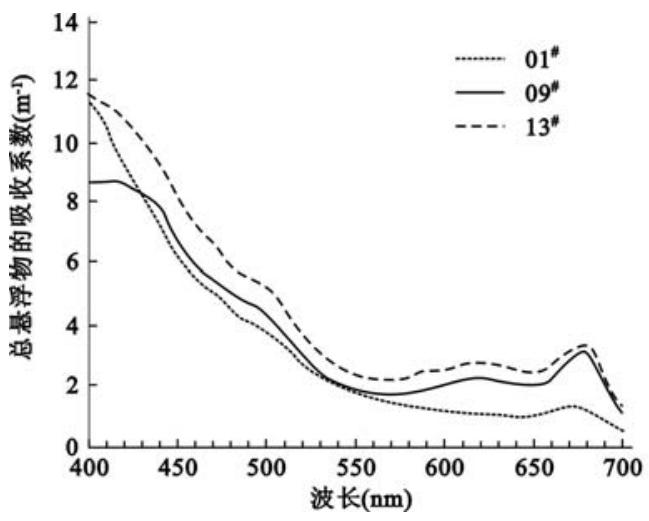

图 3 总悬浮颗粒物的吸收系数

Fig. 3 Absorption coefficients of particles matter

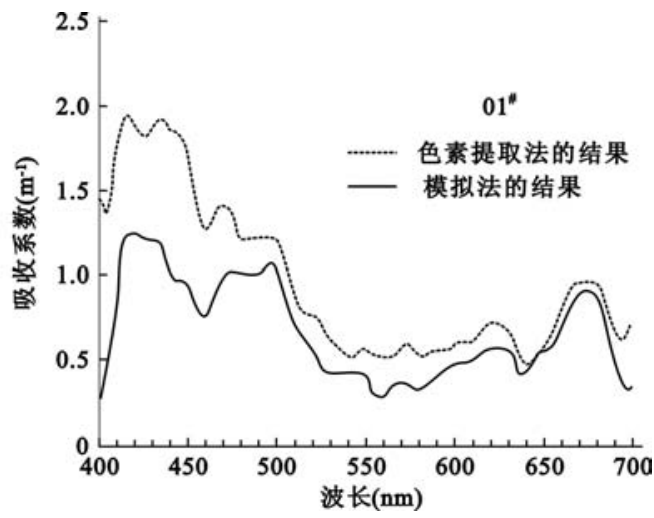

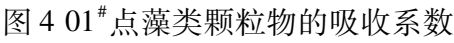

Fig. 4 Absorption coefficients of algal particles in $01^{\#}$

图 3 描述的是 $01^{\#} 、 09^{\#} 、 13^{\#}$ 样品中总悬浮颗粒物的吸收系数. $01^{\#}$ 的谱吸收系数基本呈现为随波长的增 加, 其吸收系数以负指数形式衰减, 只在 $675 \mathrm{~nm}$ 左右有一个很弱的吸收峰, 因而其谱吸收表现为非藻类颗 粒物的吸收特点, 说明在 $01^{\#}$ 点的水样中, 非藻类颗粒物所占的比重较大 ${ }^{[12]} ; 09^{\#}$ 点的谱吸收系数在 $400-$ $430 \mathrm{~nm}$ 之间变化不大,这是非藻类、藻类颗粒物共同贡献的结果,且就吸收系数而言,两者贡献基本相当; 而在 $675 \mathrm{~nm}$ 左右, 其表现出了一个较为明显的峰值, 这是由于非藻类颗粒物的吸收系数在该波段处对总吸 收系数贡献较小, 而藻类颗粒物的贡献占有明显的优势, 这也说明, 相对 01 点而言, 藻类颗粒物的比重较

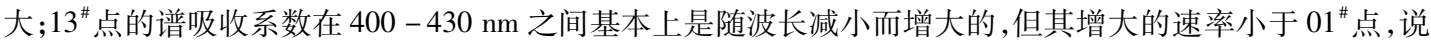
明了非藻类对总吸收系数的贡献占有优势 ${ }^{[12]}$, 但其优势不如 $01^{\#}$ 点的明显, 同时在 $675 \mathrm{~nm}$ 左右也存在一个 明显的吸收峰, 其原因在 $09^{\#}$ 点的分析中已作了说明. 这也说明了对处于富营养化阶段的大型浅水湖泊而 言, 非藻类颗粒物丰富, 基本上是总悬浮颗粒物吸收特性的主要决定者, 与海洋中悬浮颗粒物的吸收特 
性 ${ }^{[3]}$ 有着较明显的不同.

另 $01^{\#} 、 09^{\#} 、 13^{\#}$ 的有机颗粒物占总悬浮颗粒物比重分别为 $11.28 \% 、 41.91 \% 、 28.59 \%$, 这也从侧面印证 了上面的分析.

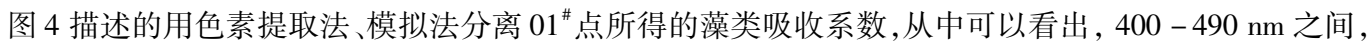
两种方法所得的结果差别较大, 在 530 - 630 nm 之间的差别次之, 其余波段的差别相对较小; $13^{\#}$ 号点二者 的差别与 $01^{\#}$ 的基本一致,但其特征不如 $01^{\#}$ 号点明显（图 6);09”点二者吻合的非常一致（图 5 ); 造成这种

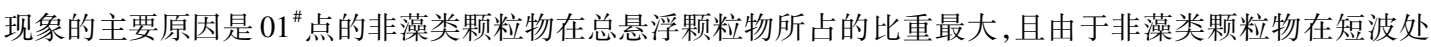
的吸收较强, 致使用色素提取法在较大程度上放大了藻类颗粒物的吸收系数; 相对于 $400-490 \mathrm{~nm}$ 来说, 非 藻类颗粒物吸收在 530 - 630 nm 得到进一步衰减,但由于藻类颗粒物中的色素在此波段吸收作用较小,因 而与藻类颗粒物相比较而言, 非藻类颗粒物贡献减小得并不厉害, 所以二者的差别依然较大; 而 490-530 $\mathrm{nm}$ 之间却由于附属色素 (如叶绿素 b、c 等) 的吸收峰的原因, 使得其非藻类颗粒物相对藻类颗粒物而言, 其 贡献相对较小, 因而其差别有所减小; 在 630-700 nm 之间由于叶绿素 a 的主吸收峰、附属色素吸收作用, 及非藻类吸收随波长加大进一步衰减, 造成藻类颗粒物的吸收贡献更大, 所以促使二者的差别最小. 在 $13^{\#}$ 点中, 由于非藻类颗粒物相对藻类颗粒物而言, 其比重较小, 因而其对总悬浮颗粒物的吸收作用也相对较 小,用色素提取法对藻类吸收作用放大的效果也就有所减小,二者的差别也随之减小, 且在上述的各个波段 之间都得到体现 (图 6). 而在 $09^{\#}$ 号点, 由于有机悬浮颗粒物的比重进一步提高, 所以非藻类颗粒物的吸收 相对藻类颗粒物而言, 其贡献甚微, 因而二者的差别最小.

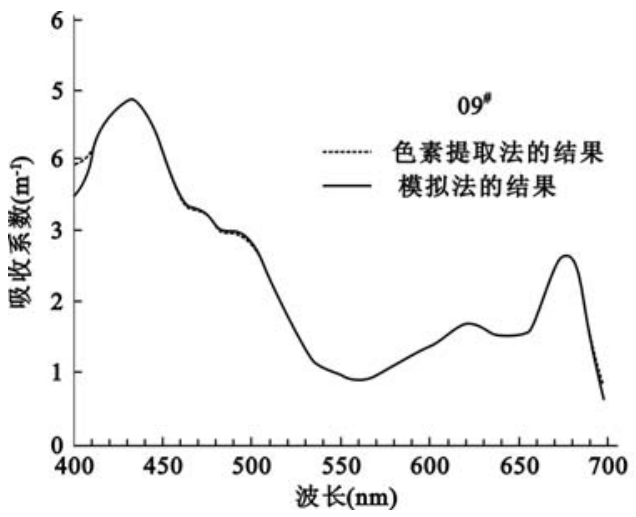

图 $509^{\#}$ 点藻类颗粒物的吸收系数

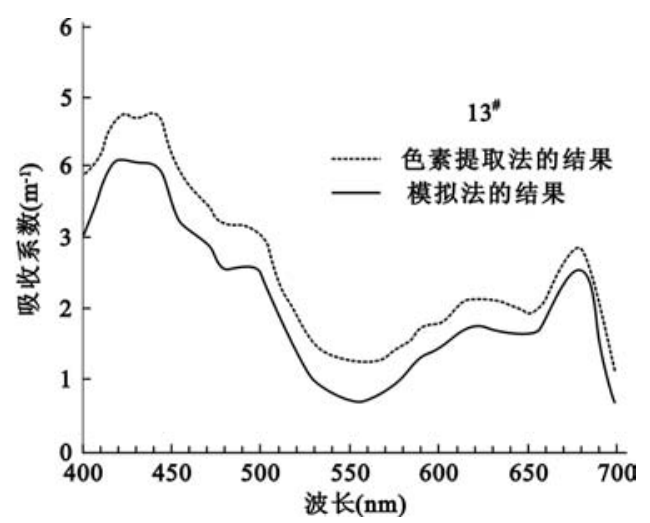

图 $613^{\#}$ 点藻类颗粒物的吸收系数

Fig. 5 Absorption coefficients of algal particles in $09^{\#}$

Fig. 6 Absorption coefficients of algal particles in $13^{\#}$

从上述中可以看出, 造成的二者的差别大小在一定程度上依赖于总悬浮颗粒物谱吸收的形状, 即藻类 颗粒物与非藻类颗粒物的比例. 当 $a_{p}$ 的谱形状显示出的藻类颗粒物吸收特征越明显,两种方法得到的结果 越相近; 反之, 则色素提取法系统放大藻类颗粒物吸收程度越大 ${ }^{[2,3,12]}$. 这也说明了在一般情况下, 对于非藻 类颗粒物来源丰富的内陆水体而言, 色素提取法并不适用于对藻类颗粒物、非藻类颗粒物吸收系数的分离.

\section{3 结论}

(1) 太湖梅梁湾区域中, 非藻类颗粒物浓度较高, 用色素提取法所得的藻类吸收系数呈现出较明显的 非藻类颗粒物的特征, 造成藻类颗粒物吸收系数有所放大,且在短波段处体现的尤为明显.

(2) 而基于光谱标准的模拟法能较好地将藻类颗粒物的吸收系数从总悬浮颗粒物吸收系数中分离出 来, 与色素提取法相比, 藻类颗粒物在 $440 、 675 \mathrm{~nm}$ 吸收峰处的吸收系数与叶绿素 $\mathrm{a}$ 的浓度相关性 $\left(R^{2}\right)$ 得到 了较为明显的提高, 分别由原来的 $0.66 、 0.75$ 提高到了 0.8964 和 0.8401 .

(3) 就色素提取法而言, 总悬浮物吸收的谱形状对藻类吸收系数的放大程度有较大的影响, 当其越接 近藻类颗粒物的吸收特征时, 则色素提取法造成的误差越小; 相反, 当其越接近非藻类颗粒物的吸收特征 
时,则色素提取法造成的误差越大.

\section{4 参考文献:}

[1] Mobley C D. Light and Water, Radiative Transfer in Nature Waters, New York: Academic Press, 1994 : 86 -98 .

[2] Cleveland J S and Perry M J. A model for partitioning particulate absorption into phytoplanktonic and detritus components. Deep-Sea Research, 1994, 41(1):197 - 221.

[3] Bricaud A and Stranmski D. Spectral absorption coefficients of living phytoplankton and nonalgal biogenous matter: A comparison between the Preu upwelling area and the Sargasso Sea. Limnol \& Oceanogr, 1990,35 (3) : :562-582.

[4] Bukata R P, Jerome J H, JainS C and Zwick H H. Optical water quality model of lake Ontario. 1: Determination of the optical cross section of organic and inorganic particulate in Lake Ontario. Appl Opt,1981,20 (9) : $1696-1703$.

[5] Kishino M M , Okami N and Ichimura S. Estimation of the spectral absorption coefficient of the phytoplankton in the sea. Bulletin of Marine Science, 1985,37(2):634-642.

[6] 曹文熙,杨耀忠, 许晓强等. 珠江口悬浮颗粒物的吸收光谱及其区域模式. 科学通报, 2003,48(17): 1876 - 1882.

[7] 张运林, 秦伯强, 杨龙元. 太湖梅梁湾悬浮颗粒物和 CDOM 的吸收特性. 生态学报, 2006(待刊).

[8] Perry M J and Porter S M. Determination of the cross-section absorption coefficient of individual phytoplankton cells buy analytical flow cytometry. Limnol \& Oceanogr,1989,34(8) : 1727 - 1738.

[9] Gallegos C L and Neals P J. Partitioning spectral absorption in Case 2 waters: Discrimination of dissolved and particulate components. Applied Optics,2002,41(21):4220 - 4233.

[10] Dupouy C, Neveux J and Andre J M. Spectral absorption coefficient of phtoposynthetically active pigments in the equatorial Pacific Ocean $\left(165^{\circ} \mathrm{E}-150^{\circ} \mathrm{W}\right)$. Deep Sea Res II ,1997,44(9) :1881 - 1906.

[11] Kirk J T O. Light and photosynthesis in acquatic ecosystems. Cambridge University Press, 1994 :72 - 78.

[12] Roesler C S and Perry M J. Modeling in situ phytoplankton absorption from total absorption spectra in productive inland marine waters. Limnol \& Oceanogr,1989,34(8):1510 - 1523.

[13] Gallegos C L. Optical water quality of a blackwater river estuary: the Lower St. Johns River, Florids, USA. Estuar. Coast Shelf $S, 2005,63: 57$ - 72 .

[14] Morrow J H. A two-component description of spectral absorption by marine particles. Limnol \& Oceanogr, $1989, \mathbf{3 4}(8): 1500-1509$.

[15] Chang G C and Dickey T D. Partitioning in situ total spectral absorption by use of moored spectral absorption-attenuation meters. Applied Optical, 1999,38(18):3876-3887.

[16] Cleveland J S and Weidemann A D. Quantifying absorption by aquatic particles: A multiple scattering correction for glass-fiber filters. Limnol \& Oceanogr,1993, 38(6) :1321 - 1327. 